\title{
Short Communication: Skeletal Maturation of Children with Sex Chromosome Abnormalities
}

\author{
M. LYNN WEBBER, MARY H. PUCK, MARION M. MARESH, WALTER B. GOAD, AND \\ ARTHUR ROBINSON ${ }^{(24)}$
}

\begin{abstract}
National Jewish Hospital and Research Center/National Asthma Center, Denver, Colorado [M.L.W., M.H.P., and A.R.J; Department of Psychiatry, University of Colorado School of Medicine, Denver, Colorado [M.H.P.]; Los Alamos Scientific Laboratories, Los Alamos, New Mexico [W.B.G.]; and Department of Pediatrics and Department of Biochemistry, Biophysics and Genetics, University of Colorado School of Medicine, Denver, Colorado [A.R.] USA
\end{abstract}

\section{Summary}

Skeletal maturity, or "bone age," is one of the several criteria used to determine developmental or physiologic age as opposed to chronologic age. The purpose of this study of skeletal maturation of children with sex chromosome abnormalities $(45, X, 47, X X X$, $47, X X Y, X$-chromosomal mosaics) and controls is $2 c$-fold: (1) to investigate if children with sex chromosome aneuploidy ascertained in an unbiased fashion differ in skeletal maturation from their siblings and other normal healthy children born in Denver, Colorado, and (2) to assess if the skeletal age standards currently in use (Greulich-Pyle; Tanner-Whitehouse) are applicable to Denver children when evaluating radiographs for skeletal maturation. Mean chronologic and skeletal age were measured. Mean differences between skeletal and chronologic age for all groups across all measures were calculated.

The 45,X females constitute the only group studied with bone ages lower than expected $(0.05>P>0.01$; two-tailed test $)$. We found no other significant differences in skeletal maturation between Denver children with sex chromosome abnormalities and their siblings or the control sample of Denver children.

Although we found the Tanner-Whitehouse standards to be more applicable for use with this population, all the Denver groups investigated yielded consistently lower bone ages than expected published norms.

This is the first documentation in a group of children with sex chromosome abnormalities, ascertained in an unbiased fashion, that, with the exception of those with a $45, X$ karyotype, bone age is not significantly different from that of the normal population.

Skeletal maturity, or "bone age," is one of several criteria used to determine developmental or physiologic age as opposed to chronologic age.

The purpose of this study of skeletal maturation of children with sex chromosome abnormalities is 2-fold: (1) to investigate if children with sex chromosome aneuploidy ascertained in an unbiased fashion differ in skeletal maturation from their siblings and other normal healthy children born in Denver, Colorado, and (2) to assess if the skeletal age standards currently in use (GreulichPyle; Tanner-Whitehouse) are applicable to Denver children when evaluating radiographs for skeletal maturation.

Although there have been studies investigating the skeletal maturation of selected $45, X$ (Turner syndrome) females $(1,3,7$, $13,17-19,22)$, little data are available on the skeletal development of individuals with other sex chromosome aberrations $(4,19)$. We know of no detailed investigations of the skeletal maturation of children with these chromosome abnormalities ascertained in an unbiased fashion.

It is well documented that $45, \mathrm{X}$ female adults have skeletal ages below published standards when untreated with estrogens at puberty $(3,19)$. Tanner et al. (19) using 27 subjects with the 47,XXY karyotype between 6-l8 years, found the period of growth for these individuals to be "greatly extended". Skeletal fusion did not occur without adrogenic hormone supplementation therapy. There are few, if any, published data on the skeletal maturation of $47, \mathrm{XXX}$ females.

\section{MATERIALS AND METHODS}

The subjects for this investigation are children involved in a longitudinal study of 51 children ( 50 white, 1 black) with various sex chromosome abnormalities (Table 1). These children were ascertained in an unbiased fashion at birth from three Denver hospitals between 1964 and 1974, and have been regularly followed with physical and psychological evaluations $(5,14-16,21)$. Karyotypically normal male and female siblings of the propositi serve as controls. Another selected group of 90 children (44 females, 46 males) from the Colorado Child Research Council (CRC) are also used as a control sample of normal healthy white children born in Denver. [See Hansman and Maresh (8) and McCammon (12) for more comprehensive developmental studies of this latter group].

Children with sex chromosome abnormalities and their siblings received biannual hand-wrist radiographs from the ages of 2 years to adolescence for determination of skeletal age. Children from the Child Research Council also received periodic hand-wrist radiographs for evaluation of skeletal development.

All roentgenograms were evaluated by Dr. Marion Maresh (23) to control for interevaluator differences. The evaluations made of the x-rays of the children with sex chromosome abnormalities and their siblings were read "blind" with respect to karyotype.

Although both studies (sex chromosome and Child Research Council) are longitudinal in design, to control for differences in number of $x$-rays per child the most recent $x$-ray taken before the age of 16 was used for comparison. Those children whose last $x$ ray was obtained before the age of 4 years were omitted from the analyses, as standards for the TW2 Carpal and radius-ulnar-small bones (RUS) ratings are not well defined below this age. If a child was receiving hormone therapy when the most recent radiograph was obtained, the last $\mathrm{x}$-ray taken before receiving supplementation was used in the analyses.

Two methods of analysis of these radiographs were used: (1) Todd's Inspectional Technique as outlined in The Radiograph Atlas of Skeletal Development of the Hand and Wrist by Greulich and Pyle (6); and (2) the Tanner and Whitehouse Method (TW2) for ratings of overall skeletal maturation (20 Bone Score), including the individual assessments of Carpal Age and RUS Age which, when combined, yield the 20 Bone Score (20).

Standards given in the Greulich and Pyle Atlas are based on the Research Series of the Brush Foundation Study consisting of upper and middle class children studied in the 1930's in Cleveland, Ohio. Mean bone ages and their S.D. at specific chronologic ages 
Table 1. Chronologic age and observed skeletal age parameters

\begin{tabular}{|c|c|c|c|c|c|c|}
\hline & Mean CA & $\begin{array}{c}\text { Greulich-Pyle } \\
\text { Mean bone age } \\
\text { (yr } \pm 1 \text { S.D.) }\end{array}$ & \multicolumn{3}{|c|}{ Tanner-Whitehouse (TW2) } & $\begin{array}{c}\text { Mean difference } \\
\text { RUS-Carpal age } \\
(\mathrm{yr} \pm 1 \text { S.D. })\end{array}$ \\
\hline $\begin{array}{c}45, X \text { and variants } \\
n=9\end{array}$ & $7.4 \pm 2.7$ & $5.4 \pm 3.0$ & $6.1 \pm 2.3$ & $6.7 \pm 2.5$ & $5.7 \pm 2.1$ & $1.0 \pm 1.1$ \\
\hline $\begin{array}{l}\text { Female mosaics } \\
\qquad n=7\end{array}$ & $8.9 \pm 2.5$ & $8.8 \pm 2.3$ & $8.8 \pm 2.1$ & $9.5 \pm 2.6$ & $8.4 \pm 1.8$ & $1.1 \pm 1.1$ \\
\hline $47, \mathrm{XXY} n=12$ & $7.0 \pm 2.8$ & $5.9 \pm 3.1$ & $6.4 \pm 3.3$ & $6.8 \pm 3.7$ & $6.3 \pm 2.9$ & $0.5 \pm 1.3$ \\
\hline $\begin{array}{l}\text { Male sibs } n=15 \\
\text { Denver controls }\end{array}$ & $10.3 \pm 2.9$ & $10.0 \pm 3.7$ & $10.1 \pm 3.5$ & $10.6 \pm 3.9$ & $9.7 \pm 3.1$ & $0.9 \pm 1.3$ \\
\hline Females $n=44$ & $7.9 \pm 2.5$ & $7.5 \pm 2.6$ & $7.8 \pm 2.7$ & $8.0 \pm 2.9$ & $7.6 \pm 2.5$ & $0.4 \pm 1.4$ \\
\hline Males $n=46$ & $8.5 \pm 2.3$ & $8.0 \pm 2.5$ & $8.3 \pm 2.5$ & $8.5 \pm 2.7$ & $8.1 \pm 2.4$ & $0.4 \pm 1.1$ \\
\hline
\end{tabular}

are given for males and females every 6 months from 1-6 years, and then every 12 months up to age 17 for males and 15 for females.

For comparison with Greulich and Pyle Standards, Standard " $Z$ " Scores were obtained for all ascertained bone ages on each child using a linear interpolation of means and standard deviations for chronologic age as outlined in their atlas (6). Mean $Z$ scores were computed across all groups, and $t$ values were obtained to determine if these mean differences varied significantly from 0 .

The standards given in the Tanner and Whitehouse Atlas are based on lower and middle class British children born in the 1950's. Tanner justifies the use of these norms on the lack of a trend toward earlier maturity in London children based on age of menarche. Tanner and Whitehouse do not specifically recommend the use of "bone age" per se, but rather set forth a measure of "maturity" based on when $50 \%$ of the population has obtained a particular maturation point between $0 \%$ and $100 \%$. They do, however, give "bone age" equivalents in their text. For purposes of this comparison study we felt justified in using them.

The S.D. for the 20 Bone Score (TW2) over the ages of 4-14 years for females and 16 years for males, are stated as \pm 1 year around the expected mean which is where bone age $=$ chronologic age (CA). The given S.D. for the individual RUS and Carpal ratings are also given as \pm 1 year over the same age range with the S.D. decreasing over the upper ages. Tanner proposes that the mean difference between Carpal and RUS is 0 years between 2-13 years of age in boys and 2-11 years in girls, with the standard deviations in both groups being \pm 1 year to \pm 0.7 year.

Therefore, observed deviations from the mean can be thought of as Standard (Z) Scores, and $t$ values were computed to determine if the sample groups differ significantly from the TannerWhitehouse norms.

\section{RESULTS}

Mean chronologic and skeletal age measures are given in Table 1. Mean differences between skeletal age and chronologic age for all groups across all measures are presented numerically in Table 2.

47, $X X X$. The eleven $47, X X X$ females between the ages of 4-13 years are not significantly different from either published standards of overall skeletal maturation. When the individual RUS and Carpal ratings of the TW2 standards are analyzed, however, this group has a significantly larger mean Carpal-CA difference than would be expected $(0.01>P>0.001$; two-tailed test) and a mean RUS-Carpal difference also reaching statistical significance $(0.01>P>0.001$; two-tailed test). This would seem to indicate a large difference in the rate of maturation between the carpal bones and the RUS bones which might be of clinical significance. These females tend to have a higher mean RUS rating than expected, although not significantly; thus, when both components are weighted into the 20 Bone Score they are found to have the expected mean skeletal age (SA).

In comparison to the group of female siblings, this group did not have any significant differences between skeletal maturation across all measures.

When compared to the Denver control sample of females, the 47, XXX females show a significant split between their Carpal and RUS ages $(0.05>P>0.01$; two-tailed test). They also tend to have lower Carpal-CA ratings $(P<0.1)$ than observed for the Denver control females.

$45, X$ and $45, X$ variants. This group of nine females [six 45,X; two $46, \mathrm{XXq}-;$ and one $45, \mathrm{X} / 46, \mathrm{Xr}(\mathrm{X})]$ between $4.5-12$ years were found to have significantly large mean differences on all measures of skeletal maturation when compared to published standards. The mean difference of -2.49 years between skeletal age and chronologic age for the Greulich-Pyle assessments reaches the greatest level of significance $(P<0.001$; two-tailed test). All difference measures using the TW2 reference for this group were found to be significant at the 0.05 level.

Using the GP assessment, this group of $45, \mathrm{X}$ females is found to have a mean SA-CA difference that is significantly different from the 11 female siblings $(0.05>P>0.01$; two-tailed test). When comparison is made between these two groups on the TW2 Bone Score, however, there is no significant difference. On the other hand, a significant difference is found between these two groups on the measure of RUS age $(0.05>P>0.01$; two-tailed test).

In comparison to the Denver control females, again the only significant difference observed in skeletal maturation is that the 45, X females have lower RUS ages than expected $(0.05>P>$ 0.01 ; two-tailed test).

Female mosaics. This group is comprised of seven females between $5-11$ years with mosaic cell lines (four $45, \mathrm{X} / 46, \mathrm{XX}$; one $46, X X / 47, X X X ;$ one $45, X / 47, X X X$; and one $45, X / 46, X X /$ $47, X X X)$. These females, who are phenotypically normal, were not found to have any significant differences in overall skeletal age from expected, using both methods of assessment and standards.

When the individual component RUS-CA of the TW2 20 Bone Score is analyzed in this group, a significant mean difference of $0.65 \pm 0.62$ years $(0.05>P>0.01$; two-tailed test) is observed; however, this result is confounded by the fact that this sample is comprised of individuals with various cell lines and no real conclusions can be drawn. This group also has a mean RUSCarpal difference that is significant when compared to the norms at the 0.05 level. When the composite scores are combined, they yield an expected mean overall skeletal age.

$47, X X Y$. This group of twelve 47,XXY males between 4-12.5 years have mean overall SA-CA differences that differ significantly from the expected for both TW2 $(0.05>P>0.01)$ and the GP $(P<0.001)$ standards. When the individual components of 
Table 2. Displacement of bone age development from standard TW2 and GP populations, expressed in units of standard deviations ${ }^{1}$

\begin{tabular}{|c|c|c|c|c|c|c|c|}
\hline & & \multicolumn{2}{|c|}{ NORMALS } & Mosaics & $47, X X Y$ & $47, X X X$ & $45, x$ \\
\hline$n=$ & & 90 & 26 & 7 & 12 & 11 & 9 \\
\hline \multirow{3}{*}{ TW2 } & $\Delta$ (RUS Age) & 0.04 & 0.36 & 0.65 & 0.20 & 0.55 & -0.85 \\
\hline & $\Delta$ (Carpal Age) & -0.36 & -0.60 & -0.53 & -0.57 & -0.87 & -1.68 \\
\hline & (RUS-Carpal) & 0.40 & 0.89 & 1.18 & 0.37 & 1.46 & 0.92 \\
\hline
\end{tabular}

' The S.D. within the populations reported vary little from unity.

the TW2 are examined, it is found that neither the RUS ratings or the Carpal ratings yield a significantly lower skeletal age than expected.

When compared to the male siblings and the male Denver control group, however, no significant differences were found for any measure of skeletal maturation.

Siblings. The 11 females siblings are between $5-13$ years of age. The 15 male siblings are between 5.5-15 years of age. Both groups have significant mean differences in overall skeletal age ratings when compared to the GP standards $(0.05>P>0.01$; two-tailed tests for both groups). No significant difference is observed for the overall skeletal age measure when compared to the TW2 standards. In the breakdown of composite ratings, the only significant finding is the difference between RUS and Carpal ratings of 0.5 years $(0.05>P>0.01$; two-tailed test) in the male siblings.

The male and female siblings are not found to have mean SACA differences varying significantly from the male and female Denver control groups on any measure.

Denver controls. This group is comprised of 44 females between 4-12 years and 46 males between $4.5-15$ years. Unlike the sibling control groups, no significant differences are found for overall skeletal maturation when compared to the published standards $(P$ $>0.05$ ). It is observed that the GP assessments yield a greater mean difference between skeletal age and chronologic age than the TW2 assessments, with use of the GP standards leading to a lower mean skeletal age for both males and females.

The males in this sample of Denver children show a significant difference between their RUS and Carpal ratings from TW2 standards, with Carpal ratings being lower $(0.05>P>0.01)$.

Female controls do not evidence any significant differences between assessed bone ages and published standards.

\section{DISCUSSION}

In assessing bone ages of Denver children with sex chromosome abnormalities ascertained in an unbiased manner and their karyotypically normal siblings, we find that the Tanner-Whitehouse standards are more applicable for use with this population. As was observed by Acheson et al. (2), use of the Greulich and Pyle norms yield almost consistently lower bone ages than the Tanner-Whitehouse assessments. The one exception is the group of mosaic females who obtained the same mean overall skeletal maturation ages by both methods. Mathiasen (11), in a study of Danish children, and Koshi et al. (10), investigating Finnish children, also found bone ages to be below expected when compared to Greulich-Pyle norms, and more closely matched the Tanner-Whitehouse standards.

It is observed that even using the TW2 standards, all groups of Denver children studied have mean overall bone ages which are consistently less than mean chronologic age.

Our data on 45,X females is in agreement with previous studies of this group $(3,19,7,13)$. The $45, X$ females are the only group studied that have mean bone ages that are lower than expected when compared to the control group of healthy Denver females including siblings.

We find no other significant differences in skeletal maturation between Denver children with sex chromosome abnormalities and their siblings or the control sample of Denver children. This is the first documentation in an unbiased group of children with sex chromosome abnormalities $(47, \mathrm{XXX}, 47, \mathrm{XXY}$, and sex chromosome mosaics) that bone age is not abnormal or significantly different from that of the normal population.

There seems to be a fairly systematic split between RUS and Carpal ratings compared to TW2 standards in these samples, with Carpal ages being lower than RUS ages.

There has been speculation that large differences between RUS and Carpal ratings may be of diagnostic significance, although there is no clear evidence of this (20). There has also been criticism that Carpal ratings contribute little to overall skeletal maturation scores and that their inclusion in overall ratings consistently lowers bone ages (9). The argument set forth is that if the addition of Carpal ratings has the effect of lowering the overall maturation scores, then these ratings should be omitted from the assessment. If this is consistently true across populations, we might assume this to be a systematic error in a normal population, and norms would need to be corrected accordingly; however, if these differences are not consistent over normal or sample groups, as observed in this study, they may in fact reflect clinically important differences. Because both systematic and variable differences may be in operation, we feel it is wiser to evaluate these differences and not discard the contribution of Carpal ratings until this discrepancy is resolved.

These samples are small. It is acknowledged that more data are needed to reach definitive conclusions; however, this group of children with sex chromosome abnormalities is unique in being ascertained in an unbiased fashion.

Without using local controls or establishing appropriateness of standard reference, our conclusions regarding children with sex chromosome anomalies would have been erroneously skewed in the direction of significantly delayed skeletal maturation. These findings emphasize the need for centers to obtain local baseline norms for bone age as well as to determine which standard method of assessing skeletal maturity most approximates their normal population. The data presented here strongly indicate that in the absence of local norms or genetically controlled comparison, assumptions utilizing skeletal maturation parameters should be cautiously made.

\section{REFERENCES AND NOTES}

1. Acheson, R. M. and Zampa, G. A.: Skeletal maturation in ovarian dysgenesis and Turner's syndrome. Lancet. $1: 917$ (1961).

2. Acheson, R. M.. Vicencio, J. H., and Fowler, G. B.: Studies in the reliability of assessing skeletal maturity from X-rays. III. Greulich-Pyle Atlas and TannerWhitehouse Methods contrasted. Hum. Biol., 38: 204 (1966).

3. Brook, C. G. D., Murset, G., Zachmann, M., and Prader, A.: Growth in children with 45,XO Turner's syndrome. Arch. Dis. Childhood, 49: 789 (1974).

4. Davies, T. S.: Bone abnormalities and XXYY. Lancet, $I$ : 92 (1970).

5. Goad, W. B., Robinson, A., and Puck. T. T.: Incidence of aneuploidy in a human population. Am. J. Hum. Genet., 28: 62 (1967).

6. Greulich, W. W. and Pyle, S. 1.: Radiographic Atlas of Skeletal Development of the Hand and Wrist. Ed. 2. (Stanford Univ. Press, Stanford, Calif., 1959).

7. Grumbach, M. M., Van Wyk, J. J., and Wilkins, L.: Chromosomal sex in gonadal dysgenesis (ovarian agenesis): relationship to male pseudohermaphrodism and theories of human sex differentiation. J. Clin. Endocrinol., 15: 1161 (1955).

8. Hansman, C. F. and Maresh, M. M.: A longitudinal study of skeletal maturation. Am. J. Dis. Child., 101: 305 (1961).

9. Johnston, F. E. and Janina, S. B.: The contribution of the carpal bones to the assessment of skeletal age. Am. J. Phys. Anthropol., 23: 349 (1965).

10. Koshi, K., Haataja, J., and Lappelanien, M.: Skeletal development of hand and 
wrist in Finnish children. Am. J. Phys. Anthropol., 19: 379 (1961).

11. Mathiasen, M. S.: Determination of bone age and recording of wrist or skeletal hand anomalies in normal children. Danish Med. Bull. 20: 80 (1973).

12. McCammon, R. W.: Human Growth and Development. (Charles C. Thomas, Springfield, Ill., 1970).

13. Mellman. W. J.: The diagnostic usefulness of skeletal maturation in an endocrine clinic. Pediatrics. 23: 530 (1959).

14. Puck. M.. Tennes. K.. Frankenburg. W.. Bryant, K.. and Robinson. A.: Early childhood development of four boys with 47, XXY karyotype. Clin. Genet., 7: 8 (1975).

15. Robinson, A.: Studies on infants and children with sex chromosomal abnormalities. In: C. Borek, C. M. Fenoglio, D. W. King (Eds.): Advances in Pathobiology, No. 6, Cancer Biology. IV. Differentiation and Carcinogenesis. pp. 214-226 (Stratton Intercontinental Medical Book Corp., New York, 1977).

16. Robinson, A., Puck, M., Pennington, B., Borelli, J., and Hudson, M.: Abnormalities of the sex chromosomes: A prospective study on randomly identified newborns. In: A. Robinson. H. A. Lubs. D. Bergsma (Eds.): Sex Chromosome Aneuploidy: Prospective Studies on Children. The National FoundationMarch of Dimes, Birth Defects: Original Article Series, Vol. XV, No. I. pp 203-24l (Alan R. Liss, Inc., New York, 1979).

17. Soyka, L. F., Ziskind, A., and Crawford, J. D.: Treatment of short stature in children and adolescents with human pituitary growth hormone, (Raben): Experience with thirty-five cases. N. Engl. J. Med., 27l: 754 (1964).

18. Stimmler, L. and Brown, G. A.: Growth hormone secretion provoked by insulin induced hypoglycaemia in children of short stature. Arch. Dis. Childhood, 42 : 232 (1967).

19. Tanner, J. M., Prader, A., Habich, H., and Ferguson-Smith, M. A.: Genes on the $\mathrm{Y}$ chromosome influencing rate of maturation in man. Skeletal age studies in children with Klinefelter's (XXY) and Turner's (XO) syndromes. Lancet, 2 141 (1959).

20. Tanner, J. M., Whitehouse, R. H., Marshall, W. M., Healy, M. J. R., and Goldstein, H.: Assessment of Skeletal Maturity and Prediction of Adult Height (TW2 Method). (Academic Press, New York, 1975).

21. Tennes, K., Puck, M., Orfanakis, D., and Robinson, A.: The early development of 17 boys with sex chromosome anomalies: A prospective study. Pediatrics, 59: 574 (1977).

22. Van der Werff ten Bosch, J. J.: Somatic Growth of the Child. p. 38 (Stenfert Kroese, Leiden, Netherlands, 1966).

23. Dr. Maresh is the former radiologist for the Child Research Council in Denver. Colorado.

24. Requests for reprints should be addressed to: Dr. Arthur Robinson, National Jewish Hospital and Research Center, 3800 E. Colfax Ave., Denver, CO 80206. 25. This research was supported in part by grant 5R01-HDI0032 from the U.S Public Health Service: by grant RR-69 from the General Clinical Research Centers Program of the Division of Research Resources, National Institutes of Health; and by The Genetic Foundation, Inc.

26. Received for publication June 22, 1981.

27. Accepted for publication October 22, 1981. 